\title{
Review on advanced diagnostic techniques for Mycobacterium species and its significance to control Tuberculosis
}

\begin{abstract}
Mycobacteria species are a group of bacteria that are responsible for tuberculosis infection which affects mainly lung, lymph glands, skin, bone disease in humans and animals worldwide. Tuberculosis (TB) is an infectious and transmissible disease mainly caused by Mycobacterium tuberculosis complex and it is one of the major public health problems worldwide that affects all age groups. Ethiopia ranks ninth among 22 high tuberculosis burden countries in world and one of the top 3 in Africa. The disease is affecting large segments of the populations in the country. It is caused by the genus of Mycobacterium which includes many pathogens known to cause serious disease in mammals. Species in Mycobacterium tuberculosis complex group are characterized by $99.95 \%$ or greater similarity at nucleotide level and posses identical 16 SrRNA sequences but differ widely in terms of their host tropisms, phenotypes and pathogenecity. The diagnosis of Mycobacterium species can be made by direct and indirect methods including clinical examination, gross and histopathological examination, Mycobacteriological culture isolation methods and biochemical tests.
\end{abstract}

These methods can be grouped as conventional mycobacterial dignosictic techniques. Other tests include immunological techniques (tuberculin skin test, interferon gamma release assays, lymphocyte proliferation assay). Furthermore, there are advanced diagnostic molecular techniques includes DNA probes, gene amplification methods, PCR, Multiplex PCR, RT-PCR, restriction fragment length polymorphism, Spoligo typing, MIRU-VNTR assay, and whole Genome sequencing. The combination of conventional, immunological and molecular techniques is the best option to develop appropriate control and prevention measures against tuberculosis in both human and animals.

Keywords: conventional diagnosis, molecular diagnosis, mycobacterium species, tuberculosis, PCR, RT-PCR, MIRU-VNTR assay
Volume 6 Issue 3 - 2018

\author{
Eskendir Kedir,' Gezahegne Mamo, ${ }^{2}$ Kibeb \\ Legesse, ${ }^{2}$ Asmelash Tassew ${ }^{3}$ \\ 'Addis Ababa University, Ethiopia \\ ${ }^{2}$ Department of Biomedical Sciences, College of Veterinary \\ Medicine and Agriculture, Addis Ababa University, Ethiopia \\ ${ }^{3}$ Department of Animal Science \& Technology, Raya University, \\ Ethiopia
}

\begin{abstract}
Correspondence: Asmelash Tassew, Department of Animal Science \& Technology, College of Agriculture and Natural Resources, Raya University, P O Box 92, Maychew, Ethiopia, Tel 0025 I9 I328 2043, Email asmelash21@yahoo.com
\end{abstract}

Received: October 19,2018 | Published: May 10, 2018

\section{Introduction}

Tuberculosis (TB) is an infectious and transmissible disease caused by Mycobacterium tuberculosis complex ${ }^{1}$ and one of the major public health problems worldwide that affects all age groups. In 2012 alone, there were 8.6million new cases and 1.3million deaths globally and remains a global emergency health problem in $21 \mathrm{st}$ century and continued to be leading cause of death globally., ${ }^{2,3}$ Today, HIV infection is the major risk factor for developing TB disease in individuals with latent tuberculosis infection. ${ }^{4}$ It is caused by the genus of Mycobacterium which includes many pathogens known to cause serious disease in mammals. ${ }^{5}$

Despite the different species tropisms, the Mycobacterium tuberculosis complex is characterized by $99.95 \%$ or greater similarity at nucleotide level and posses identical 16 SrRNA sequence. ${ }^{6,7}$

In 2014, the World Health assembly passed agreement resolution approving the new post-2015 Global TB Strategy. The strategy aims to end the global TB epidemic, and has set targets to reduce TB deaths by $95 \%$ and to cut new cases by $90 \%$ between 2015 and 2035 . It also attempt to ensure that no family is burdened with catastrophic expenses due to TB. Ethiopia is one of the high tuberculosis endemic countries in the world where the disease is leading cause of mortality and morbidity. Ethiopia ranks ninth among 22 high tuberculosis burden countries and one of the top 3 in Africa. The disease is affecting large segments of the populations in the country. ${ }^{3}$

In Ethiopia, the endemic nature of bovine tuberculosis in domestic animals has long been reported and most recent studies also showed that BTB is endemic mainly in cattle in large parts of the country. ${ }^{8-12}$ Cattle are considered to be the true hosts of M. bovis. In addition other species of livestock including goat, camel, sheep, and swine are known to be infected by M. bovis in Ethiopia.

For effective diagnosis of TB there is a growing perception with no single method is sufficient for detecting all tuberculosis patients. ${ }^{13}$ The diagnosis of mycobacterium species can be made by direct and indirect methods, in which clinical, post mortem (animals), histopathological, immunological, bacteriological and molecular methods. ${ }^{14}$ The rapid rise of drug-resistant TB has further complicated TB diagnosis and drug susceptibility tests are essential to monitor the spread of resistant TB strains, and ensure that patients are given effective treatment. ${ }^{15}$ 
Advanced immunological and molecular diagnostic methods are important for early confirmation of the diagnosis of TB in case of paucibacillary and extra-pulmonary forms and for differentiate between Mycobacterium tuberculosis complex and non tuberculosis mycobacteria and where strain differentiation is not possible by conventional approach.

Therefore, the objectives of this paper are to give concise review on advanced diagnostic techniques of Mycobacterium species and its significance to control tuberculosis.

\section{Literature review}

Mycobacteria are a group of bacteria that are responsible for many infections in humans and animals worldwide. This group can be classified into three main groups due to their growth rate like the fastgrowers and the slow-growers. The major groups of these organisms that pose the biggest threat are the M. tuberculosis complexes which can cause tuberculosis disease that did not found in the environment include $M$, tuberculosis, $M$. bovis, $M$. africanum and M. microti. The second group is $M$. leprae which is the causative agent of leprosy. The last group constitutes the non tuberculous mycobacteria, which are all the environmental mycobacteria that may cause various disease resembling tuberculosis, lymphadenitis, skin disease, or disseminated disease. ${ }^{16}$

Tuberculosis lesions in cattle are most often found in organs rich in reticulo endothelial tissue, particularly the lungs and associated lymph nodes, as well as the liver. ${ }^{17}$ Other studies conducted on naturally and experimentally infected cattle demonstrated that lesions are most commonly present in the lower respiratory tract; however the upper respiratory tract and associated tissues may also display disease in many cases. ${ }^{18,19}$ Although tubercles are not pathognomonic of Bovine tuberculosis, the presence of clinical signs of the disease is directly associated to their distribution and quantity. ${ }^{20}$

In cattle, it is usually characterized by the formation of nodular granulomoas known as tubercles and commonly defined as chronic debilitating occasionally progressive. ${ }^{21}$ Bovine tuberculosis occur worldwide and zoontic importance of disease and production loss due to chronic nature. ${ }^{22} M$.bovis in human is the most frequent cause of zoonotic TB which is clinically indistinguishable from TB caused by $M$. tuberculosis especially intestinal TB in children consuming of unpasteurized milk..$^{23}$

\section{Properties of the Mycobacterium species}

These bacteria are known as tubercle bacilli because they produce characteristic lesions called tubercles. ${ }^{24}$ By culture isolation of mycobacterium, the ideal medium should be able to support rapid and luxuriant growth, and allow the determination of its characteristic features like colony morphology, growth rate and pigment production. ${ }^{25}$

The luxuriant growth of $M$. tuberculosis on glycerol containing media, giving the characteristic rough, tough and buff colonies is known as eugenic while the growth of M. avium on media containing glycerol is also described as eugenic whereas M. bovis has sparse, thin growth on glycerol containing media that is called dysgenic, however, grows well on pyruvate-containing media without glycerol. ${ }^{25}$

\section{Diseases caused by Mycobacterium species}

The Mycobacterium genus comprises more than 120 different species and is distributed worldwide. Among them are pathogenic species which can cause serious diseases in humans and animals is typical mycobacteria that cause tuberculosis in animals and humans. Mycobacterium tuberculosis complex causing tuberculosis are M. tuberculosis, M. africanum, M. bovis, M. canettii, M. microti, M. caprae, M. orygis, and M. pinnipedii, \& M. leprae which can cause Hansen's disease (leprosy). Non tuberculous Mycobacteria, are Mycobacteria Other Than Tuberculosis or Atypical Mycobacteria can produce localized disease in the lungs, lymph glands, skin, wounds or bone. ${ }^{26}$

\section{Epdemiological status of Tuberculosis}

The global distribution of tuberculosis cases is skewed heavily toward low income and emergence economies. $95 \%$ of all cases and $99 \%$ of deaths due to tuberculosis occur in developing countries with the greatest burden in Sub Saharan Africa and South East Asia., ${ }^{1,3}$ Ethiopia is one of the highest tuberculosis endemic countries in the world where the disease is leading cause of mortality and morbidity. Ethiopia ranks ninth among 22 high tuberculosis burden countries in world and one of the top 3 in Africa. The disease is affecting large segments of the populations in the country. ${ }^{3,27}$

Despite disease under reporting in developing countries, there is sufficient evidence to indicate not only that the prevalence of disease is higher in the developing nations, but also that in the absence of national control and eradication programmes, it is increasing worldwide, particularly in Africa, Asia and Latin America.$^{28}$ According to the World Animal Health Information Database (WAHID) of the World Organisation for Animal Health (OIE), 70 countries reported bovine tuberculosis cases in their cattle populations in 2010, and 49 countries in $2011 .^{28,29}$

Additional challenges to TB diagnosis is that specific epidemiological factors like HIV infection is thought to be a major contributor to the increase in TB incidence across the world. ${ }^{6}$ The estimated $9 \%$ of adults globally with newly diagnosed TB are HIV positive, but this rate is $31 \%$ in Africa and diagnosis can also be more difficult in children. ${ }^{30}$

Slightly high TB infection in general population of cattle in most part of Ethiopia and cattle are considered to be the true hosts of $M$. bovis as shown in Table 1 \& Table 2. Bovine tuberculosis has been reported in many surroundings of Ethiopia according to southern Ethiopia, around Dilla study showed that prevalence of BTB in cattle was low as compared to prevalence of BTB in intensive dairy farms in central Ethiopia. ${ }^{12}$ This high prevalence of BTB indicates a high TB infection potential in the general population of cattle in Jimma town and its surroundings. ${ }^{31}$ Similarly, the study in Afar region revealed a moderately high prevalence of bovine tuberculosis in cattle and the presence of epidemiological risk factors for infection and transmission among cattle and communities have very close contact with their animals and depend entirely on their livestock for subsistence through consumption of raw milk and other animal products, have high risk for human infection. ${ }^{11}$ Another research in Northern Ethiopia around Mekele town showed that the communities in the study area lack 
awareness on routes of transmission of BTB and this may allow circulation of the agent between the communities and animals. ${ }^{32}$ Very recent study conducted in cattle slaughtered at Gambella municipal Abattoir revealed a moderate high prevalence of BTB. ${ }^{33}$

Table I Tuberculosis infection in general population of cattle in most part of Ethiopia

\begin{tabular}{llll}
\hline Author & Study place & Host & Disease prevalence \\
\hline $\begin{array}{l}\text { Ameni \& Wudie,et } \\
\text { al. }{ }^{9}\end{array}$ & Adama & Cattle & $5.20 \%$ \\
Demlash et al. $^{10}$ & Adama & Cattle & $4.20 \%$ \\
Demelash et al. $^{10}$ & $\begin{array}{l}\text { Adama another } \\
\text { abattoir }\end{array}$ & Cattle & $24.70 \%$ \\
Tigre et al. $^{31}$ & Jimma & Cattle & $5.40 \%$ \\
${\text { Temsegen } \mathrm{M}^{41}}^{31}$ & Bishoftu & Cattle & $11.10 \%$ \\
Jemberu et al. $^{33}$ & Gambella & Cattle & $13.20 \%$ \\
Fikru et al. ${ }^{32}$ & Mekele & Cattle & $11.30 \%$ \\
\hline
\end{tabular}

Table 2 Tuberculosis infection in general population of cattle in most part of Ethiopia

\begin{tabular}{|c|c|c|c|}
\hline Author & Study place & Host & $\begin{array}{l}\text { Disease } \\
\text { prevalence }\end{array}$ \\
\hline $\begin{array}{l}\text { Ameni \&Wudie } \\
\text { et al. }{ }^{\text {? }}\end{array}$ & Adama & Cattle & $5.20 \%$ \\
\hline Demlash et al. ${ }^{10}$ & Adama & Cattle & $4.20 \%$ \\
\hline Demelash et al. ${ }^{10}$ & $\begin{array}{l}\text { Adama another } \\
\text { abattoir }\end{array}$ & Cattle & $24.70 \%$ \\
\hline Tigre et al. ${ }^{31}$ & Jimma & Cattle & $5.40 \%$ \\
\hline Temsegen $\mathrm{M}^{41}$ & Bishoftu & Cattle & $11.10 \%$ \\
\hline Jemberu et al. ${ }^{33}$ & Gambella & Cattle & $13.20 \%$ \\
\hline Fikru et al. ${ }^{32}$ & Mekele & cattle & $11.30 \%$ \\
\hline
\end{tabular}

Diagnosis of Tuberculosis in humans and animals

\section{Conventional diagnostic techniques}

Clinical examinations: Are the primary tuberculosis diagnostic methods which help for person suspects of pulmonary tuberculosis when presenting with persistent cough for 2 weeks or more detection in human tuberculosis. ${ }^{34}$ In human patients chest radiography is useful for differential diagnosis of pulmonary disease and with negative sputum smears interpreted with conjunction with presenting signs and symptoms which of reading of X-Ray. ${ }^{35}$ The presence of $M$. bovis in clinical and post-mortem specimens may be demonstrated by examination of stained smears or tissue sections and confirmed by cultivation of the organism on primary isolation medium that preferably sterile use of sample containers are not contaminated by environmental mycobacteria may result in the failure to identify M. bovis infection due to the rapid growth of the environmental mycobacteria. ${ }^{21}$

Ziehl-neelsen staining/ZNS or acid fast staining/AFS: It is a special bacteriological stain used to identify acid-fast organisms, like Mycobacterium contain large amounts of lipid substances within their cell walls, called mycolic acids which resist staining by ordinary methods such as Gram stain and the most widely used rapid diagnostic test, simple and inexpensive. Acid fast bacteria microscopy the most practical and fastest technique in establishing a diagnosis of pulmonary $\mathrm{TB}$, especially in developing countries, where most of the TB cases live. ${ }^{36}$

Acid-fast bacilli (AFB) can be identified with Ziehl-Neelsen (ZN) staining method in resource limited settings. ${ }^{4,37}$ Smeared directly onto the slides without any processing and subjected to $\mathrm{ZN}$ staining and it's sensitivity and specificity of direct $\mathrm{ZN}$ microscopy is low when compared to the culture method limitations is that the threshold for detection of acid-fast bacilli in sputum samples under optimal conditions is between $10^{4}$ and $10^{5}$ bacilli per $\mathrm{ml},{ }^{38}$ For pulmonary TB the centrifugation of extra pulmonary tuberculosis samples and fluorochrome staining with ultraviolet microscopy increases the sensitivity of microscopy by $10 \%$ of ZN staining. ${ }^{39}$

Abattoir survey and histopathology: Study in different abattoirs of Ethiopia based on detailed meat inspection and microscopic examination, there were presence of bovine TB in Nazareth Municipality abattoir reported ${ }^{10}$ and in Nekemte. ${ }^{40}$ In similar studies especially in Jimma abattoir ${ }^{31}$ and Bishoftu Elfora export abattoir, paratuberculosis in cattle ${ }^{41}$ bovine tuberculosis was prevalent in cattle slaughtered at Gambella municipal Abattoir with moderately high prevalence as shown below. ${ }^{33}$

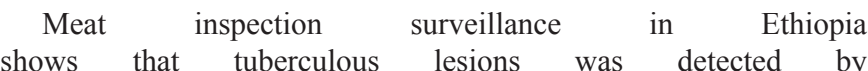
sitive PCR (IS6110) was positive in animals with tuberculous lesions and in animal without lesions. ${ }^{42}$

Detection of BTB among the slaughtered animals indicates the presence of BTB in the Egyptian animal husbandry with relevance to human zoonoses, proper implementation of meat inspection procedures during slaughtering with public awareness are important to control BTB in Egypt. ${ }^{43}$

Mycobacterial culture: Most of mycobacterial culture media fall into egg-potato-base media and agar-base media. The most popular eggbased media are the Lowenstein-Jensen buffered egg potato medium and among the agar based media, Middle brook 7H10, Middle brook $7 \mathrm{H} 11$, and Dubose oleic-albumin agar are recommended and the advantages of egg-based media are long shelf life up to 1 year when refrigerated and the low cost of preparation. ${ }^{44}$

Isolation of Mycobacterium species from clinical samples by culture is the "gold standard" for a definitive diagnosis of EPTB. Culture methods are much more sensitive because fewer bacilli (10-100bacilli $/ \mathrm{ml}$ of concentrated material) can be detected and provides the necessary isolates for conventional drug susceptibility test and species identification. ${ }^{45}$

By culture isolation of mycobacterium, the ideal medium should be able to support rapid and luxuriant growth, and allow the determination of its characteristic features like colony morphology, growth rate and pigment production. ${ }^{25}$

The luxuriant growth of M. tuberculosis on glycerol containing media, giving the characteristic rough, tough and buff colonies is known as eugenic while the growth of M. avium on media containing glycerol is also described as eugenic whereas $M$. bovis has sparse, thin growth on glycerol containing media that is called dysgenic, however, grows well on pyruvate-containing media without glycerol. ${ }^{25}$

For the recovery of Mycobacterium, which culture can be stored 
for a long period of time before being used for molecular and other techniques egg-based media such as Lowenstein Jensen media have been used for a long time. ${ }^{46}$

When grown on LJ medium, the media must be incubated for a significant length of time, usually 4 weeks, due to the slow doubling time of M. tuberculosis (15-20h) compared with other bacteria and appears as brown, granular colonies. Thus, wider use of LJ culture has limited potential to accelerate TB diagnosis. ${ }^{47}$

\section{Biochemical tests}

Nitrate reduction test: It is valuable for identification of some Mycobacteria like $M$.tuberculosis, M. kansasii, m. szulgai that posses similar characteristics of colony morphology, growth rate and pigmentation due to quantitatively in their abilities most rapid growth and nitrate the ability to produce the enzyme reductase and $M$. bovis nitrate reduction are negative. ${ }^{48}$

Thiophene 2 carboxylic hydrazide (TCH): It is used to distinguish M. bovis susceptible to low concentration ( 1 to $5 \mathrm{~g} / \mathrm{ml})$ of $\mathrm{TCH}$ from Mycobacterium tuberculosis complex and other non chromogenic slowly growing mycobacteria and $M$. tuberculosis complex can be differentiated from $M$. bovis by its ability to grow in the presence of $\mathrm{TCH}$, a property shared by most mycobacteria species except for $M$. bovis. ${ }^{49}$

Pyrazinamindase test: The enzyme pyrazinamidase hydrolyses pyrazinamide to pyrazinoic acid and is detected in culture medium by the addition of ferrous ammonium sulfate, the formation of pink ferrous pyrazinoic and complex indicates a positive test which useful in separating $M$.tuberculosis from M. bovis. ${ }^{50}$

\section{Immunological tests}

Tuberculin skin test (TST): The TST based on a delayed type hypersensitivity to mycobacterial tuberculoprotein, is the standard ante mortem test both in human and cattle. It is convenient, cost effective method for assessing cell mediated responses to a variety of antigens and it is "gold standard" for diagnostic screening for detection of new or asymptomatic MTC infection..$^{51}$

TST is most accurate and preferred type of skin test when those cattle infected with $M$. tuberculosis are exposed to a purified protein derivative, a mixture of antigens shared by several mycobacteria that gives rise to a skin reaction. In this type of reaction, T cells, sensitized by prior infection, are deposited to the skin where the antigen was injected and release lymphokines. The ultimate result is local in swelling of the skin through local vasodilatation, edema, fibrin deposition, and recruitment of other inflammatory cells to the area. ${ }^{36,52}$

The immunological diagnosis of tuberculosis which consists of intradermic inoculation of the purified protein derivative (PPD) and in measuring 48 to 72 hours post inoculation delayed hypersensitivity which measure the cell mediated immune response against the 75 tubercle bacilli. ${ }^{52,53}$

The immune suppressor in human patients that allows lower tuberculin skin test response due to considering the effect of pathogenic mycobacteria to which the animal may have been exposed. environmental Mycobacterium, such as HIV/AIDs, diabetes mellitus, Due to time consuming, logistics, requires long incubation time and cancer diseases, protein energy malnutrition and taking steroid drugs is relatively expensive, it is not been accepted to inter laboratory for longer period of time and BCG vaccination in most developing comparisons. ${ }^{56}$ countries, including Ethiopia. ${ }^{54}$
Tuberculin skin test has been widely used as a screening test in many countries and has contributed substantially to generate valuable epidemiological information on infection trends and on the magnitude of disease..$^{55}$

Single intradermal tuberculintest (SIDT): The intradermal injection of $0.1 \mathrm{ml}$ of bovine tuberculin purified protein derivative (PPD) into a skin fold at the base of the tail or into the cervical fold and the subsequent detection of swelling as a result of delayed hypersensitivity. The reaction is read between 48 and 96hours after injection with a preference for 48-72hours for maximum sensitivity and at 96 hours for maximum specificity. The positive reaction constitutes a diffuse swelling at the site of injection. ${ }^{21,52}$

The main disadvantage of the SID test is its lack of specificity and the number of reactor lesion occur. Mammalian tuberculin is not sufficiently specific to differentiate between reactions due to infection with $M$. bovis and infection with $M$. avium, $M$. tuberculosis and $M$. paratuberculosis including vaccination and failure to detect cases of minimal sensitivity, in old cows and in cows which have recently calved as well as in early infection, in some cattle in an unresponsive state, referred to as anergy which is developed due to excess antigen or immunosuppression which in turn caused by non specific factors such as malnutrition and stress..$^{21,52}$

Comparative intradermal tuberculin test (CIDT): Two sites on the mid neck, $10-12 \mathrm{~cm}$ apart, are shaved and the thickness is measured in millimeters with caliper before the injection of tuberculin. In the CIDT test, $0.1 \mathrm{ml}$ of Purified Protein Derivatives from Mycobacterium avium (PPD-A) and $0.1 \mathrm{ml}$ of Purified Protein Derivatives from Mycobacterium bovis (PPD-B) are injected intradermally into separate clipped sites on the side of the neck. Care must be taken in placing the injection as varied from place to place in the skin. After 72 hours the thickness of the skin at the sites is measured again. ${ }^{8,32}$

When the change in skin thickness is greater at the PPD-A injection site, the result is considered negative for BTB. When the change in skin thickness increased at both sites, the difference between the two changes is considered. Thus, if the increased in the skin thickness at the injection site for the bovine (B) is greater than the increase in the skin thickness at the injection site at the avian (A) and (B-A), is less than $1 \mathrm{~mm}$, between 1 and $4 \mathrm{~mm}$, or a $4 \mathrm{~mm}$ and above, the result is classified as negative, doubtful, or positive for BTB, respectively and the animal with the evidence of infection is termed as reactor. The comparative test is used to differentiate between animals infected with $M$. bovis and those responding to bovine tuberculin as a result of exposure to other Mycobacterium. This sensitization can be attributed to the antigenic cross reactivity among mycobacterial species and related genera like Nocardia. ${ }^{21}$

Lymphocyte proliferation assay: The assay compares the reactivity of peripheral blood lymphocytes to tuberculin PPD-B and PPD-A and results analyzed by PPD-B result minus PPD-A can be obtained. From peripheral whole blood sample purified lymphocytes can be done. The test determined increase specificity of assay by removing response to lymphocytes to non cross reactive antigens associated with non

In in vitro assay compares the reactivity of peripheral blood 
lymphocytes to tuberculin PPD-B and PPD-A, can be performed on whole blood or purified lymphocytes from peripheral blood samples which endeavors to increase specificity of the assay by removing the response of lymphocytes to non-specific or cross reactive antigens associated with non-pathogenic species of mycobacteria to which the animal may have been exposed. Results are usually analyzed as the value obtained in response to PPD-B minus the value obtained in response to PPD-A. The assay has scientific value, but is not used for routine diagnosis because the test is time-consuming and the logistics and laboratory execution are complicated, meaning it requires long incubation times and the use of radioactive nucleotides. As with the IFN- test, the lymphocyte proliferation assay should be performed shortly after blood is collected. The test is relatively expensive and has not been subjected to inter laboratory comparisons. ${ }^{5,52,57}$

\section{Interferon gamma release assays (IGRAS): IGRAS} are more sensitive and specific immunological assays designed for immunological diagnosis of Mycobacterium tuberculosis which a substitute of tuberculin skin test. ${ }^{53}$ IGRAS positive gives an indication of an infection, but cannot distinguish similarly to tuberculin skin test between active tuberculosis from latent infection. The Quantiferon Tuberculosis Gold in the Tube is an Interferon gamma release assays that measure the amount of interferon gamma secreted following re stimulation, and many laboratories provide to clinicians the results in $\mathrm{UL} / \mathrm{ml} .^{58}$ In animals that are aggressive difficult to handle, such as excitable cattle or other bovidae, the advantage of the IFN- test over the skin test is that the animals need be captured only once. The IFN- test is used for serial testing (to enhance specificity) and parallel testing (to enhance sensitivity). The advantages of the IFN- assay are its increased sensitivity, the possibility of more rapid repeat testing and no need for a second visit to the farm and more objective test procedures. The limitations of IFN comprise a reduced specificity, high logistical demands (culture start is required within 24 hours after blood sampling), an increased likelihood of non specific response in young animals [Owing to natural killer cell activity] and its high cost. $^{21,30}$

Molecular techniquesDNA probes: Such techniques are often categorized as advanced diagnostic techniques of Mycobacterium species because they do have modern and sophisticated lab facilities and activities. The method is very easy to perform, and no special instrumentation is needed. The probes have been evaluated extensively in clinical practice and are rapid, sensitive and specific..$^{59,60}$

This technique is used for specific identification and rapidly confirming the diagnosis with in 1 or 2 days compared to much longer time required with classical biochemical tests of Mycobacterium tuberculosis which is not very sensitive and need more than 10000 organisms in the specimen for positivity. ${ }^{61-64}$

DNA probes for the identity of mycobacterial isolates based on information about specific gene sequences well defined oligonucleotide probes for identification of $M$. tuberculosis, M. avium and several other mycobacteria used for rapid confirming the diagnosis within 1 to 2 days with gene probes as compared to much longer time required with classical biochemical tests.

16s rRNA sequencing based probes: Ribosomal DNA finger printing for development of probes as well as gene amplification assays for various types of mycobacterial species including M. tuberculosis, $M$. leprae, M. avium, M. gardonae etc used ribosomal RNA gene region which probes that target rRNA, ribosomal DNA, spacer and flanking sequences and probes are commercially available..$^{61-64}$

The faecal of cattle were tested all culture positive samples were positive in PCR assay. Three universal primers were used to sequence the amplified fragments of the 16s rRNA gene of various species of mycobacteria. When the nucleotide sequences were analysed, a deletion was detected in the sequence of the fast-growing species. An oligonucleotide probe $(\mathrm{P})$ directed to this region was synthesized and hybridized directly with total RNA of various mycobacterial strains in a dot-spot assay. The probe detected M. paratuberculosis, some other slow growing mycobacteria of the $M$. avium-intracellulare (MAI) complex and one atypical strain, $M$. gordonae. To increase the sensitivity of the probe, a 413-bp fragment of the 16s rRNA gene of M. paratuberculosis between $\mathrm{P}$ and a second oligo nucleotide primer was amplified and hybridised with a $M$. paratuberculusis or $M$. auium-specific probe. When faecal samples of cattle were tested, all culture-positive samples were positive in the PCR assay. ${ }^{65}$

Gene amplification methods: For amplification of specific gene regions followed by hybridization with species specific probes ${ }^{66,67}$ and sequencing and RFLP analysis for confirmation of the identity of isolates this techniques may also used. ${ }^{68}$

Gene amplification techniques are highly sensitive and under optimum conditions which adequately standardized, evaluated and precautions for avoiding the contamination can play a very useful role in early confirmation of diagnosis in paucibacillary extra-pulmonary forms of tuberculosis detect 1-10 organisms. ${ }^{69,70}$

Polymerase chain reaction (PCR): PCR is a molecular technique that allows sequences of DNA to be amplified and make thousands of copies from very few DNA of $M$. tuberculosis and small concentration of DNA in a sample can be rapidly identified by PCR. ${ }^{71}$

PCR is a powerful tool that is used in a wide Varity of diagnostic procedures and used to detect the presence of genetic material, DNA that is unique and specific to an organism of interest. PCR works by amplifying a portion of DNA that is specific for the organism. This product can be easily visualized using laboratory procedures. The PCR test is very sensitive and can detect the presence of organism when present at very low level. ${ }^{21}$

The method consists of PCR amplification of mycobacterial DNA with genus-specific primers and sequencing of the amplicons become the gold standard for definitive identification of mycobacterial species by comparison of the nucleotide sequence with reference sequences and also direct detection of mycobacterial species that cannot be grown on conventional laboratory culture media. ${ }^{72,73}$

The advantages of amplification technique over culture are higher sensitivity, as it can detect as few as 1-10 organisms in clinical specimens at least in research conditions. These tests can be performed on the stored samples, and provide etiological diagnosis in short span of 6-8 hours. ${ }^{27}$

With advance of molecular diagnosis various PCR methods in diverse clinical specimens have been introduced to identify Mycobacterium tuberculosis complex more easily and quickly compare to conventional techniques, and that has several advantages over culture, including ,confirmation of the presence of Mycotuberculosis within 3 days as compared to 6 weeks with the traditional microbiological 
methods, puacibacilary nature of specimen and extensive differential Region of deletion PCR: This method is advanced approach to diagnosis in extra pulmonary Tuberculosis that require a rapid, determine the subspecies of MTC isolates and to differentiate them sensitive and specific diagnosis needed for in developing countries from clinically important MOTB species that help proven beneficial and it is commercially available., ${ }^{5,64}$ Additional advent-ages of PCR in the management of Mycobacterium collections and may be applied over conventional methods include its high sensitive, performances in for practical clinical and epidemiological use due to rapid, simple, few hours and depending on the assay design, ability to differentiate and reliable PCR-based Mycobacterium tuberculosis complex typing between Mycobacterium tuberculosis Complex and Mycobacterial method that makes use of chromosomal region-of-difference deletion species. ${ }^{34}$

Observing Mycobacterium Tubeculosis Complex in tissues or smears using ZN staining or Fluorescence method allows faster diagnosis. Unfortunately, these methods are in sensitive and non specific. This is cause for development of new and sensitive diagnostic techniques like PCR. ${ }^{5}$

Key Mycobacterial targets for PCR amplifications are the insertion sequence IS0, IS6110, $65 \mathrm{KD}$ (kilodalton) heat shock protein, $38 \mathrm{KD}$ protein and ribosomal RNA. This is found in almost all members in high number in most strains of the Mycobacterium tuberculosis Complex. ${ }^{5}$

PCR methods allow direct identification of Mycobacterium tuberculosis complex and can detects less than 10 bacteria in a clinical specimen. PCR sensitivity ranges from $70-90 \%$ compared to the result of culture and its specifty varies between 90 and $95 \%$. In smear positive cases, the sensitivity of PCR is greater than $95 \%$ but in smear negative cases, it is only 50 to $60 \% .^{13} \mathrm{~A}$ recent study performed in Bangladesh reported that PCR was positive in $94.74 \%$ cases and the specificity was $100 \%$ using IS6110 that enables a rapid technique for the diagnosis of pulmonary TB. ${ }^{74}$

For the diagnosis of bovine tuberculosis, PCR is used to identify $M$. bovis in tissue collected at necropsy from animals suspected of being infected with bovine tuberculosis. PCR is the only used on tissue that have histological (microscopic) evidence compatible with bovine tuberculosis. The result can typically be obtained within 7 days and are classified as either positive or negative. A positive test obtained on PCR is highly suggestive that the animal is infected with bovine tuberculosis. PCR has been widely evaluated for the detection of Mycobacterium tuberculosis complex in clinical samples, mainly sputum in human patients and has recently been used for the diagnosis of tuberculosis in animals. ${ }^{75}$ Multiplex PCR: The multiplex PCR comprised of genus-specific primers targeting hsp 65 gene, Mycobacterium tuberculosis complex specific primer targeting cfp10 (Rv3875, esxB ) region and Mycobacterium avium complexspecific primer pairs targeting 16S-23S Internal Transcribed Spacer sequences. Two hundred and thirty-five samples from 145 clinically suspected cases of tuberculosis were processed for the detection of Mycobacterial infections, the multiplex PCR developed had an analytical sensitivity of 10fg (3-4 cells) of mycobacterial DNA. The multiplex PCR test showed the highest (77.24\%) detection rate, while ZN smear examination had the lowest $(20 \%)$ detection rate, which was bettered by L-J culture (34.4\%) methods. ${ }^{7}$ Real time PCR (RT-PCR): Real time PCR used for confirming the diagnosis and monitoring the progress for rapid and specific detection of $M$. tuberculosis in the clinical specimens. ${ }^{77}$ Implementing RT-PCR recent polycentric study of a large number of non respiratory specimens reported an overall sensitivity of $81 \%{ }^{78}$ The sensitivities with respiratory specimens, with the exception of a sensational $98 \%$ reported in the first assessment. ${ }^{79}$ loci by which amplify within the loci 16S rRNA, Rv0577, IS1561', Rv1510, Rv1970, Rv3877/8, and Rv3120) were run in separate but simultaneous reactions and each primer pair either specifically amplified a DNA fragment of a unique size or failed, depending upon the source mycobacterial DNA. The pattern of amplification products from all of the reactions, visualized by agarose gel electrophoresis, allowed immediate identification either as MTC composed of $M$. tuberculosis (or M. africanum subtype II), M. africanum subtype I, $M$. bovis, M. bovis BCG, M. caprae, M. microti, or "M. canettii" or as a Mycobacterium other than MTC (MOTB). ${ }^{80}$

The RD9 deletion typing was carried out on isolates that showed band for M. tuberculosis complex by genus typing was tested each sample in a separate PCR tube. Primers directed against the RD9 were used to generate a deletion profile that would allow species identification of the isolate by using of flanking primers of RD9 that contains 2030 base pair (bp) gene segment of M. tuberculosis and PCR Analysis revealed that RD9 is absent in M. bovis M. microti and M. africanum ${ }^{33,81}$ and Primers used for RD9 typing and the size of PCR product size expected in the presence or absence of the respective region of difference. ${ }^{33,82}$

Restriction fragment length polymorphism (RFLP): This type of test, using IS6110-based restriction fragment length polymorphism (RFLP), has proven useful in phylogenetic studies of TB bacilli. RFLP DNA fingerprinting is the gold standard for strain typing in mycobacteriology. This method of genotyping has been standardized in order to increase the inter- and intra-laboratory comparability, so that it could be used for sub speciation of Mycobacterium tuberculosis. ${ }^{83}$ It is considered as a gold standard for the molecular typing of $\mathrm{M}$. tuberculosis due to its high discriminative power and reproducibility. It can also be used for outbreaks identification and can facilitate contact tracing of tuberculosis. ${ }^{13}$ However, this technique requires large amount of DNA and is therefore restricted to the mycobacterial cultures which take around 20 to 40 days to obtain sufficient DNA needed and for the combined process of probe labeling, DNA fragmentation, electrophoresis, blotting, hybridization, washing and auto-radiograph. Moreover, this technique is also technically demanding, slow, cumbersome, expensive and requires sophisticated analysis soft ware for result analysis. ${ }^{13,25}$ Roughly 1 week is needed before the results can be interpreted. The disadvantages of RFLP genotyping are that a large cell mass is required and comparison is difficult since the results are band patterns, which are hard to convert into digital formats. ${ }^{84}$

Spoligo typing: Spoligotyping is a PCR-based method for the simultaneous detection and typing of $M$. tuberculosis strains. Prior culturing of the bacteria is needed, with a sensitivity of $96 \%$ and a specificity of $98 \%$, the results can be obtained from a M. tuberculosis culture within 1 day $^{75}$ and it's the clinical usefulness of spoligotyping is determined by its rapidity, both in detecting causative bacteria and in providing epidemiologic information on strain identities that implementing would be useful in the surveillance of TB transmission and in interventions to prevent further spread of disease. ${ }^{33,75}$

Citation: Kedir E, Mamo G, Legesse K, et al. Review on advanced diagnostic techniques for Mycobacterium species and its significance to control Tuberculosis. J Bacteriol Mycol Open Access. 20 18;6(3): I68-177. DOI: I0.15406/jbmoa.2018.06.00198 
Spoligotyping also called spacer oligonucleotides typing is a novel or new method for simultaneously detection and typing of mycobacterium tuberculosis complex bacteria, has been recently developed. ${ }^{85}$ This method is based on polymerase chain reaction amplification of highly polymorphic direct repeat locus in the $M$. tuberculosis genome. The direct repeat region in M. bovis BCG contains direct repeat sequences of 36 base pairs, which is interspersed by the non-repetitive DNA spacers of 35-41 base pairs in length. Other Mycobacterium tuberculosis complex strains contain one or more IS6110 elements in direct repeat region. ${ }^{13}$

Spoligotyping applied to culture is simple, robust and highly reproducible. ${ }^{86}$ Results can be obtained from a M. tuberculosis culture within one day. Thus the clinical usefulness is determined by its rapidity, both in detecting causative bacteria and in providing epidemiologic information on strain identities. It can also be useful for identification of outbreak and can facilitate contact tracing of tuberculosis. PCR based methods are available as diagnostic and confirmatory test for tuberculosis and are expected to detect as low as 1 to 10 organisms. ${ }^{13,75}$

Implementation of such a method in a clinic setting would be useful in surveillance of tuberculosis transmission and intervention to prevent further spread of this disease. ${ }^{75}$ The specificity and sensitivity of this technique has been found to be 98 and $96 \%$, respectively with the clinical samples..$^{13}$ One of the clearest advantages of Spoligotyping over IS6110 RFLP typing is that, in principle, spoligotyping can be used simultaneously for the detection and typing of Mycobacterium tuberculosis complex bacteria in one assay and requires viable organisms. ${ }^{86}$

Mycobacterial interspersed repetitive units-variable number tandem repeat (MIRU-VNTR) typing: Mycobacterial interspersed repetitive units-variable number tandem repeat (MIRU-VNTR) typing rely on PCR-amplification using primers specific for the flanking regions of the VNTRs and on the determination of the sizes of the amplicons, after electrophoretic migration. As the length of the repeat units is known, these sizes reflect the numbers of the amplified MIRU-VNTR copies. The result is a numerical code, corresponding to the repeat number in each VNTR locus. Such numerical genotypes are intrinsically portable and are thus particularly convenient for both intra- and inter-laboratory comparative studies Initial VNTR typing systems for MTBC strains made use of very limited sets of loci including exact tandem repeats (ETR), mycobacterial interspersed repetitive units (MIRUs) and sets of Queen's University Belfast (QUB) VNTRs). ${ }^{87}$ MIRU-VNTR 24-loci typing has been proposed for international standardization based on analysis of the clonal stability and evolutionary rates of MIRU-VNTR markers in the genetic lineage of tubercle bacilli collected worldwide. ${ }^{88}$ In addition, the method has been improved as high-speed automated genotyping system with the use of multiplex PCRs for the target MIRU-VNTR loci on a fluorescence-based DNA analyzer with computerized automation of the genotyping. ${ }^{88}$

Whole genome sequencing: Whole genome sequencing has been shown to provide a rapid and comprehensive view of the genotype of the organism; it provides the highest resolution when investigating transmission events in possible outbreak scenarios. ${ }^{89}$

Next generation sequencing, important in establishing improvement by showing that fewer tuberculosis cases were clustering indicating tuberculosis transmission between individuals that the value of tuberculosis programmatic changes in reducing transmission country improvements in TB control through early diagnosis, effective treatment and improved infection, control may be masked by new migration of TB cases for primarily identifying weaknesses in institutional infection control procedures which lead to new TB cases. ${ }^{90}$

\section{Conclusion and recommendations}

Proper and definitive Diagnosis of tuberculosis is a critical component in the control of Mycobacterial infections in human and animals. The diagnostic methods can be categorized as conventional and advanced immunological and molecular methods, each having their own merits and drawbacks in term of their application and efficiency.

Molecular diagnostic techniques of Mycobacterium species have advantages than conventional methods due to their sensitivity and specificity, being less complicated, less time consuming, expensive and not cost effective than the conventional methods for early confirmation of the diagnosis of tuberculosis both in humans and animals. ${ }^{91-99}$ Molecular methods used for differentiate between $M$. tuberculosis complex and nontuberculous mycobacteria and also applicable for culture negative samples where strain identification is not possible by conventional approach. So that combinations of conventional, immunological and molecular techniques are the best diagnostic tools of TB. Knowing the available advanced and promising tuberculosis diagnostic techniques can detect and differentiate the causative mycobacterial species and help early confirmation of the diagnosis which is useful to design an appropriate control measures in the national TB-control programmes.

Based on the above concluding remarks, the following recommendations are forwarded:

I. Conventional microbiological, immunological and molecular methods should be used in combination based on their detection capacity and needs introduction in identification of the causative strains of mycobacterium species.

II. In order to overcome the limitations of specificity and sensitivity of TB diagnostic techniques in clinical settings, more advanced and reliable immunological and molecular techniques should be introduced to developing countries like Ethiopia.

\section{Acknowledgements}

None.

\section{Conflict of interest}

Authors declare that there is no conflict of interest.

\section{References}

1. Namukwaya E, Nakwagala FN, Mulekya. Predictors of treatment failure among pulmonary tuberculosis patients in Mulago hospital, Uganda. Afr Health Sci. 2011;11(Suppl 1):S105-S111.

2. Swarnlata P, Shoba K, Khublani TK. A review on TB and its advance diagnostic techniques. International Journal of Pharma and Bio Sciences. 2011;2:535-545

3. WHO. Global Tuberculosis Report 2014. Geneva; 2014.

4. WHO. Guidelines for intensified tuberculosis case-finding and isoniazid 
preventive therapy for people living with HIV in resource-constrained settings. Geneva, Switzerland; 2011.

5. Palomino JC, Laao SC, Ritacco V. Tuberculosis 2007: from basic science to patient care. Bourcillier Kamps, Belgium, 2007:53-680.

6. Dye C, Watt CJ, Bleed DM, et al. Evolution of tuberculosis control and prospects for reducing tuberculosis incidence, prevalence, and deaths globally. J Am Med Assoc. 2005;293(22):2765-2775.

7. Smith NH, Kremer K, Inwald J, et al. Ecotypes of Mycobacterium tuberculosis complex. J Therory Biol. 2006;239(2):220-225.

8. Ameni G, Vordemeier M, Firdessa R, et al. Mycobacterium tuberculosis infection in grazing cattle in central Ethiopia. Veterinary $J$ of Lancet Infect Dis. 2010;5:415-430.

9. Ameni G, Wudie A.Preliminary study on bovine tuberculosis in Nazareth municipality abattoir of central Ethiopia. Bull Anim Heal Prod Afr. 2003;51:125-132.

10. Demelash B, Inangolet F, Oloya J, et al. Prevalence of bovine tuberculosis in Ethiopian slaughter cattle based on post-mortem examination. Trop Anim Health Prod. 2009;41(5):755-765.

11. Gezahegne M, Fekadu A, Yalelet W, et al. Bovine tuberculosis and its associated risk factors in pastoral and agro-pastoral cattle herds of Afar Region, Northeast Ethiopia. Journal of Veterinary Medicine and Animal Health. 2013;5(6):171-179.

12. Gebremedhin R, Gebremedhin G, Gobena A. Assessment of bovine tuberculosis and its risk factors in cattle and humans, at and around Dilla town, southern Ethiopia. Animal and Veterinary Sciences. 2014;2(4):94 100

13. Sharma R, Gupta V. Spoligotyping for the Detection of Mycobacterium tuberculosis complexbacteria. Asian J Biochem. 2010;6:29-37.

14. Ramos DF, Silva PE, Dellagostin OA. Diagnosis of bovine tuberculosis: review of main techniques. Braz J Biol. 2015;75(4):830-837.

15. Sharma SK, Mohan A. Multidrug-resistant tuberculosis: a menace that threatens to destabilize tuberculosis control. Chest. 2006; 130(1):261272.

16. O'Brien RJ, Geiter LJ, Snider DE. The epidemiology of nontuberculous mycobacterial diseases in the United States. Results from a national survey. Am Rev Respir Dis. 1987;135(5):1007-1014.

17. Corner L, Melville L, McCubbin K, et al. Efficiency of inspection procedures for detection of tuberculous lesions in cattle. Aust Vet $J$. 1990;67(11):389-392.

18. Neill SD, Cassidy J, Hanna J, et al. Detection of Mycobacterium bovis infection in skin test-negative cattle with an assay for bovine interferon gamma. Vet Rec. 1994;135(6):134-135.

19. Rodgers JD, Connery NL, McNair J, et al. Experimentalexposure of cattle to a precise aerosolized challenge of Mycobacterium bovis: A novel model to study bovine tuberculosis. Tuberculosis. 2007;87(5):405-414.

20. Domenech De la, Goodchild R, Vordemeier AT, et al. Ante mortem diagnosis in cattle: A review of the tuberculin tests, g-interferon assay and other ancillary diagnostic techniques. Res Vet Sci. 2006;81(2):190-210.

21. http://www.oie.int/fileadmin/Home/eng/Healthstandards/tahm/2.04.07 BOVINE_TB.pdf

22. Quinne PJ, Markey BK. Concise review of veterinary Microbiology. UK: Black well Publishing; 2003. p. 34-37.

23. Ojo O, Sheehan S, Corcoran GD, et al. Mycobacterium bovis strains causing smear-positive human Tuberculosis, Southwest Ireland. Emerg Infect Dis. 2008;14(12):1931-1934

24. Chesteras M. Tuberculosis in Children and Young Adults: 1943.
25. Patterson J, Grooms D. Diagnosis of bovine tuberculosis: Gross necropsy,histopathology and acid fast staining. Michigan State University Extension: 2000;35:1-2.

26. Churchill LE, Brown E, Wallace BA, et al. Infections Due to Nontuberculous Mycobacteria Other than Mycobacterium aviumintracellularei. Principles and Practices of Infectious Diseases. 2010;2:3191-3198

27. World Health Organization. Global tuberculosis control: epidemiology, strategy, financing. WHO report Publication, Geneva; 2009.

28. Thoen CO, Lobue PA, Enarson DA, et al. Tuberculosis: a re-emerging disease of animals and humans. Veterinaria Italiana. 2009;45(1):135181.

29. Majoor CJ, Magis-Escurra C, van Ingen J, et al. Epidemiology of Mycobacterium bovis disease in humans, the Netherlands, 1993-2007. Emerg Infect Dis. 2011;17(3):457-463.

30. Corbett EL, Watt CJ, Walker N, et al. The growing burden of tuberculosis: global trends and interactions with the HIV epidemic. Arch Intern Med. 2003;163(9):1009-1021.

31. Tigre W, Alemayehu G, Abetu T, et al. Preliminary study on the pidemiology of bovine tuberculosis in Jimma town and its surroundings, Southwestern Ethiopia. African Journal of Microbiology Research. 2012;6(11):2591-2597.

32. Fikre Z, Gebremedhin R, Gebretsadik B, et al. Prevalence of bovine tuberculosis and assessment of Cattle owners' awareness on its public health implication in and around Mekelle, Northern Ethiopia. Jouranal of veterinary Medicine and Animal health. 2014;6(6):159-167.

33. Jemberu A, Gezahegne M, Gobena A, et al. Molecular Epidemiology of Bovine Tuberculosis in Cattle and its Public Health Implications in Gambella Town and its Surroundings, Gambella Regional State, Ethiopia. Global Journal of Medical Research: F Diseases. Global Journals Inc. 2016;15(5):2249-4618.

34. Federal Ministry of Health. TB, Leprosy and TB/HIV prevention and control program manual. 4th ed. Addis Ababa, Ethiopia; 2008.

35. Asnake M, Felke D. Sensitivity of chest X-rays and their relations to sputum results in the diagnosis of pulmonary TB in Hosanna. Ethiop. $J$ Health Dev. 2008;14(2):199-204.

36. Huebner RE, Schein MF, Bass JB. The tuberculin skin test. Clin Infect Dis. 1993;17:968-975.

37. Schramm B, Hewison C, Bonte L, et al. Field evaluation of a simple fluorescence method for detection of viable Mycobacterium tuberculosis in sputum specimens during treatment follow-up. $J$ Clin Microbiol. 2012;50(8):2788-2790.

38. Morello JA, Granato PA,Wilson ME, et al. Laboratorymanual and workbook in microbiology: applications to patient care. McGraw-Hill Higher Education, Boston; 2006.

39. Menzies D. Using Tests for Latent Tuberculous Infection to Diagnose Active Tuberculosis: Can We Eat Our Cake and Have It Too? Ann Intern Med. 2008;148(5):398-399.

40. Tigre W, Gudeta T, Regassa F. Preliminary study on Bovine Tuberculosis in Nekemte municipality abattoir, Western Ethiopia. Bull Anim Heal Prod Afr. 2010;58(3):323-327.

41. Mohammed T. Investigation on the occurrence and pathology of Paratuberculosis (Johne's disase) in apparently healthy cattle slaughtered at Elfora export abattoir Bishoftu, Ethiopia; 2014

42. Shityaye JE, Gtahun B, Alemayehu T, et al. A prevalence study of bovine tuberculosis by using abattoir meat inspection and tuberculin skin testing data, histopathological and IS6110 PCR examination of tissues with tuberculous lesions in cattle in Ethiopia; 2006. 
43. Youssef AI, Ahmed AM. Bovine tuberculosis survey based on meat inspection and microscopic examination in central city abattoir in Ismailia, Egypt and its hazards to the abattoir workers. International Food Research Journal. 2014;21(2):577-582.

44. Fauci S, Braunwald E, Dennis L, et al. Harrison's Principles of Internal Medicine. 17th ed. USA: McGraw-Hill Companies; 2008. p. 1543-1571.

45. Davies PD, Pai M. The diagnosis and misdiagnosis of tuberculosis. The international journal of tuberculosis and lung disease. The official journal of the International Union against Tuberculosis and Lung Disease. 2008;12(11):1226-1234.

46. Siddiqi SH. Recent advances in the diagnosis of tuberculosis: an overview. Med Sci. 1999;7(1):31-36.

47. van Cleeff MR, Kivihya-Ndugga L, Githui W, et al. A comprehensive study of the efficiency of the routine pulmonary tuberculosis diagnostic process in Nairobi. Int J Tuberc Lung Dis. 2003;7(2):186-189.

48. Virtanen S. A study of nitrate reduction by mycobcteria. Acta Tuberic Scand. 1960;48:1-119.

49. Vestal AL, Kubica GP. Differential identification of mycobacteria III. Use of thiacetazone, thiophen-2-carbohyalac acid hydrazide and triphenyltetrazolium chloride. Scan J Respir Dis. 1967;48:142-148.

50. Wayne G. Simple pyrazinamidase and urease tests for routine identification of mycobacteria. Rev Respir Dis. 1974;109(1):147-171.

51. Katial HK, Seth TP, Brannan J, et al. Cell-mediated immune response to tuberculosis antigens: Comparison of skin testing and in whole- blood culture. Cl and diagn lab Immuno. 2001;8:329-345.

52. Radostits O, Blood D, Gray C. Veterinary Medicine, a Text Book of the Diseasesof cattle, sheep, Pigs, Goats and Houses. 8thed, London: Ballier Tindals; 2007:830-838.

53. Lecouvet F, Irenge L, Vandercam B, et al. The etiologic diagnosis of infectious discitis is improved by amplification based DNA analysis. Arthritis Rheumatol. 2004;50(9):2985-2994.

54. Legesse M, Ameni G, Mamo G, et al. Community based Cross Sectional Survey of Tuberculosis in Afar Pastoralists, Ethiopia,Using QuantiFERON-TB Gold in Tube and tuberculin Skin test. BMC infect Dis. 2011;11:89.

55. Harstad I, Heldal E, Steinshamn SL, et al. Tuberculosis Screening and follow up of asylum seekers in Norway. A cohort study. BMC Public Health. 2009;9:141.

56. Lilenbaum W, Fonseca L. The use of enzyme linked immune sorbent assay as a complementary tool for bovine Tuberculosis in Brazil. Braz. $J$ Microbiol. 2005;168:167-168.

57. Regassa A, Tassew A, Amenu K, et al. A cross sectional study on bovine tuberculosis in Hawassa town and its surrounding Southern Ethiopia. Trop An Health Prod. 2010;42(5):915-920.

58. Delogu G, Zumbo A, Fadda G. Microbiological and Immunological diagnosis of Tuberculosis Spondylodiscitis. Eur Rev Med. Pharmacol Sci. 2012;1(2):73-78.

59. Lebrun L, Espinasse F, Poveda JD, et al. Evaluation of nonradioactive DNA probes for identification of mycobacteria. $J$ Clin Microbiol. 1992;30:2476-278.

60. Evans KD, Nakasone AS, Sutherland PA, et al. Identification of Mycobacterium tuberculosis and Mycobacterium avium-intracellulare directly from primary BACTEC cultures by using acridinium-esterlabeled DNA probes. J Clin Microbiol. 1992;30(9):2427-2431.

61. McFadden JJ, Kunze Z, Seechum P. DNA probes for detection and identification. In: McFadden J, editor. Molecular Biology of the Mycobacteria. Surrey University Press, UK; 1990:139-172.

62. Kaminski DA, Hardy DJ. Selective utilization of DNA probes for identification of Mycobacterium species on the basis of cord formation in primary BACTEC cultures. J Clin Microbiol. 1995;33(6):1548-1550.

63. Sharma RK, Katoch K, Shivannavar CT. Comparisons of sensitivity of probes targeting RNA versus DNA in leprosy cases. Indian $J$ Med Microbiol. 1996;14:99-104.

64. Katoch VM, Sharma VD. Advances in the diagnosis of mycobacterial diseases. Indian J Med Microbiol. 1997;15:49-55.

65. Vander JWD, Glessen A, Eger J, et al. Amplification of $16 \mathrm{~s}$ rRNA sequences to detect Mycobacterium paratuberculosis. J Med Microbiol. 1996;36:255-263.

66. De Beenhouwer H, Liang Z, De Rijk P, et al. Detection and identification of mycobacteria by DNA amplification and oligonucleotide specific capture plate hybridization. J Clin Microbiol. 1995;33(11):2994-2998.

67. Suffys PN, da Silva Rocha A, de Oliveira M, et al. Rapid identification of mycobacteria to the species level using INNOLIP Amycobacteria, a reserve hybridization assay. J Clin Microbiol. 2001;39(12):4472-4482.

68. Telenti A, Marchesi F, Balz M, et al. Rapid identification of mycobacteria to the species level by polymerase chain reaction and restriction enzyme analysis. J Clin Microbiol. 1993;31(2):175-178.

69. Forbes BA, Hicks KE. Direct detection of Mycobacterium tuberculosis in respiratory specimens in a clinical laboratory by polymerase chain reaction. J Clin Microbiol. 1993;31(7):1688-1694.

70. Verma A, Rattan A, Tyagi JS. Development of a 23S rRNA based PCR assay for the detection of mycobacteria. Indian $J$ Biochem Biophys 1994;31(4):288-294.

71. Ramachandran R, Paramasivan $\mathrm{CN}$. What is new in the diagnosis of tuberculosis? Part I; techniques for diagnosis of tuberculosis. Indian J Tuberc. 2003;50:133-141.

72. Springer B, Tortoli E, Richter I, et al. Mycobacterium interjectum, a new species isolated from a patient with chronic lymphadenitis. $J$ Clin Microbiol. 1993:31(12):3083-3089.

73. Springer B, Tortoli E, Richter I, et al. Mycobacterium conspicuum sp. nov, a new species isolated from patients with disseminated infections. $J$ Clin Microbiol. 1995;33(11):2805-2811.

74. Hasan MM, Hossain MA, Paul SK, et al. Evaluation of PCR with culture for the diagnosis of pulmonary tuberculosis. Mymensingh $\mathrm{Med}$ J. 2012;21:399-403.

75. Gori A, Bandera A, Marchetti G, et al. Spoligotying and Mycrobacterium tuberculosis. Global Veterinaria. 2005;5(5):255-258.

76. Gopinath K, Singh S. Multiplex PCR assay for simultaneous detection and differentiation of Mycobacterium tuberculosis, Mycobacterium avium complexes and other Mycobacterial species directly from clinical specimens. J Appl Microbiol. 2009;107(2):425-445.

77. Soini H, Agha SA, El-Fiky A, et al. Comparison of Amplicor and 32 kiloDalton PCR for detection of Mycobacterium tuberculosis from sputum specimens. J Clin Microbiol. 1996;34(7):1829-1830.

78. Tortoli E, Russo C, Piersimoni C, et al. Clinical validation of Xpert MTB/RIF for the diagnosis of extrapulmonary tuberculosis. Eur Respir J. 2012;40(2):442-447.

79. Boehme CC, Nabeta P, Hillemann D, et al. Rapid molecular detection of tuberculosis and rifampin resistance. $N$ Engl J Med. 2010;363(11):10051015 
80. Huard RC, Lazzarini LC, Butler WR, et al. PCR-based method to differentiate the subspecies of the Mycobacterium tuberculosis complex on the basis of genomic deletions. J Clin Microbiol. 2003;41(4):16371650 .

81. Gordon SV, Brosch R, Billault A, et al. Identification of variable regions in the genomes of tubercle bacilli using bacterial artificial chromosome arrays. Mol Microbiol. 1999;32(3):643-655.

82. Deresa B, Conraths FJ, Ameni G. Abattoir-based study on the epidemiology of caprine tuberculosis in Ethiopia using conventional and moleculartools. Acta Vet Scand. 2013;55:15.

83. Bifani P, Kurepina N, Mathema B, et al. Genotyping of Mycobacterium tuberculosis clinical isolates using IS6110-based restriction fragment length polymorphism analysis. Methods Mol Biol. 2009;551:173-188.

84. Ogbaini-Emovon E. Current trends in the laboratory diagnosis of tuberculosis. Benin J Postgrad Med. 2009;11:79-90.

85. Kamerbeek J, Schouls L, Kolk A, et al. Simultaneous Detection and Strain Differentiation of Mycobacterium tuberculosis for Diagnosis and Epidemiology. J Clin Microbiol. 1997;35(4):907-914.

86. Van Soolingen D. Molecular Epidemiology of Tuberculosis and other Mycobacterial infections: main methodologies and achievements. Journal of Veterinary Diagnostic Investigation. 2008;249(1):1-26.

87. Supply P, Mazars E, Lesjean S, et al. Variable human minisatellite-like regions in the Mycobacterium tuberculosis genome. Mol Microbiol. 2000;36(3):762-771.

88. Supply P, Allix C, Lesjean S, et al. Proposal for standardization of optimized mycobacterial interspersed repetitive unit-variable-number tandem repeat typing of Mycobacterium tuberculosis. J Clin Microbiol. 2006;44(12):4498-4510.

89. Catherine A, Amber C, Jason A, et al. Clinical use of whole genome sequencing for Mycobacterium tuberculosis. Irish Veterinary Journal. $2016 ; 14(16)$

90. Schuback S, Rüsch-Gerdes S, Supply P, et al. Wholegenome sequencing versus traditional genotyping for investigation of a Mycobacterium tuberculosis outbreak: a longitudinal molecular epidemiological study.

\section{PLoS Med. 2013;10:1001387.}

91. Duffey PS, Guthertz LS, Evans GC. Improved rapid identification of mycobacteria by combining solid phase extraction with high performance liquid chromatographic analysis of BACTEC cultures. J Clin Microbiol. 1996;34(8):1939-43.

92. Fauci S, Braunwald E, Dennis L, et al. Harrison's Principles of Internal Medicine. 17th ed. USA: McGraw-Hill Companies; 2011. p. 1543-1571.

93. Gingeras TR, Ghandour G, Wang E, et al. Simultaneous genotyping and species identification using hybridization pattern recognition analysis of generic Mycobacterium DNA arrays. Genome Res. 1998;8(5):435-448.

94. Huebner RE, Good RC, Tokars JI. Current practices in mycobacteriology: results of a survey of state public health laboratories. J Clin Microbiol. 1993;31(4):771-775.

95. Roetzer A, Diel R, Kohl TA, et al. Type mycobacterium assay for identification of mycobacterial species isolated from human clinical specimens by using liquid medium. J Clin Microbiol. 2002;40:30763078 .

96. Singh KK, Muralidhar M, Kumar A, et al. Comparison of in house PCR with Conventional techniques for the detection opf Mycobacterium tuberculosis DNA in granulomatous lymphoidenopathy. J Clin Pathol. 2000;53(5):355-361.

97. Troesch A, Nguyen H, Miyada CG, et al. Mycobacterium species identification and rifampin resistance testing with high-density DNA probe arrays. J Clin Microbiol. 1999;37(1):49-55.

98. WHO. Global Tuberculosis Report. World Health Organization, Geneva; 2012.

99. WHO. Fluorescent Light-Emitting Diode (LED) Microscopy for Diagnosis of Tuberculosis: Policy Statement. World Health Organization, Geneva, Sweeden; 2011. 